\title{
Engineering of Biomimetic Hair-Flow Sensor Arrays Dedicated to High-Resolution Flow Field Measurements
}

\author{
A.M.K. Dagamseh, C.M. Bruinink, H. Droogendijk, R.J. Wiegerink, T.S.J. Lammerink, and G.J.M. Krijnen \\ $\mathrm{MESA}+$ research institute for Nanotechnology \\ University of Twente \\ Enschede, The Netherlands \\ a.m.k.dagamseh@ewi.utwente.nl
}

\begin{abstract}
This paper addresses the latest developments in biomimetic hair-flow sensors towards sensitive high-density arrays. Improving the electrodes design of the hair sensor, using Silicon-on-Insulator (SOI) wafer technology, has resulted in the ability to measure small capacitance changes as caused by minute rotations of single-hair sensors. The detection limit, as measured in a bandwidth of $3 \mathrm{kHz}$, was about $1 \mathrm{~mm} / \mathrm{s}$ air-flow amplitude, an enhancement of $52 \%$ in comparison to the previous hair-sensor array design. The directivity pattern was improved now closely resembling a figure of eight. These sensors open possibilities for high-resolution flow pattern observations.
\end{abstract}

\section{INTRODUCTION}

The biomimetic approach deals with systems inspired by or based upon examples as found in nature. Flow-sensors based on hairs, as found on arthropods, notably on spiders and crickets, are among the most sensitive sensory systems appearing in nature. Crickets are thought to be able to perceive flow signals at thermal noise levels and, using canopies of hairs, to discern flow phenomena at high-resolution. The sensing hairs of crickets [1] and the ciliar-based lateral line system of fish [2] are examples of sensor array systems used to detect flow patterns in air and water, respectively.

A large hair-sensor-array residing on the cerci of crickets forms the sensing part of the cricket's escape mechanism e.g. when exposed to spider-attacks. Air movement due to an approaching predator causes the cricket to turn rapidly away from the stimulus [3]. The large numbers of hairs, array density, hair's mechanical properties, their directionality and the accompanying neural system, result in a system capable of detecting and localizing predators $[4,5]$.

Recently, Micro Electro Mechanical Systems technology (MEMS) has been exploited to fabricate mechanical sensors and to drive sensor miniaturization. Bio-inspired sensor designs have the promise of better performance, robustness and higher accuracy when compared with traditionally engineered designs. However, this promise can only be realized when the sensor interfacing does not deteriorate these added values. In this paper, we show the latest advancements in interfacing and fabrication of our artificial hair sensors. The renewed hair sensor design enables improved sensor directivity and reduced detection limit compared with the previous hair sensor design.

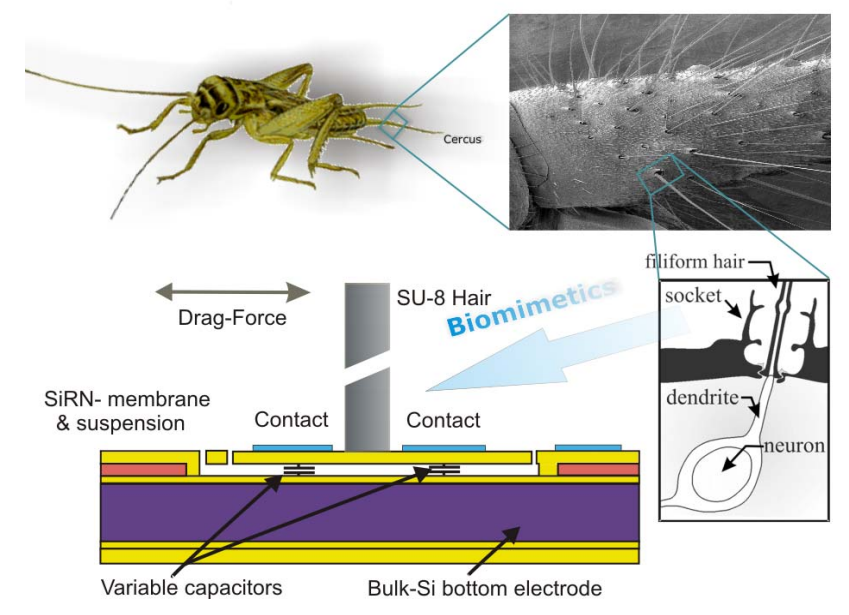

Figure 1. Artificial hair geometry and its biological source of inspiration.

\section{ARTIFICIAL HAIR SENSOR}

\section{A. Sensing principle}

Fig. 1 represents the structure of the mechano-receptive sensory-hair with its source of inspiration. The artificial hair flow-sensor, fabricated using MEMS surface micromachined technology, is formed using a suspended silicon nitride membrane with up to $1 \mathrm{~mm}$ long SU-8 hair on top. The electrodes deposited on top of the membrane form capacitors with a common underlying electrode, namely the silicon 
substrate. Due to the viscous drag torque acting on the hair, the membrane tilts and in consequence to that the capacitors, on both halves of the sensor, change equally but oppositely. In combination with two out-of-phase voltage sources (carrier signals) the changes in the capacitance are converted to a voltage signal, modulating the carrier amplitude (AM signal). A synchronous demodulation technique, which consists of multiplier followed by a lowpass filter, is used to recover the original air flow signal from the AM signal. These capacitive changes, representing the air flow surrounding the hair, are measured differentially reducing disturbances that are common to both sides of the sensor.

\section{B. Fabrication}

The previous artificial hair flow-sensor fabrication process starts with the deposition of a $200 \mathrm{~nm}$ thin silicon nitride layer on a highly conductive silicon wafer followed by a $600 \mathrm{~nm}$ polysilicon sacrificial layer. The bottom silicon forms the common electrode of the integrated capacitors whereas the polysilicon layer determines the capacitor gap. A $1 \mu \mathrm{m}$ thick silicon rich nitride layer is deposited and patterned forming the membrane and spring structures. The upper capacitor electrodes are formed by sputtering and etching a $100 \mathrm{~nm}$-thick aluminum layer on top of the membrane.

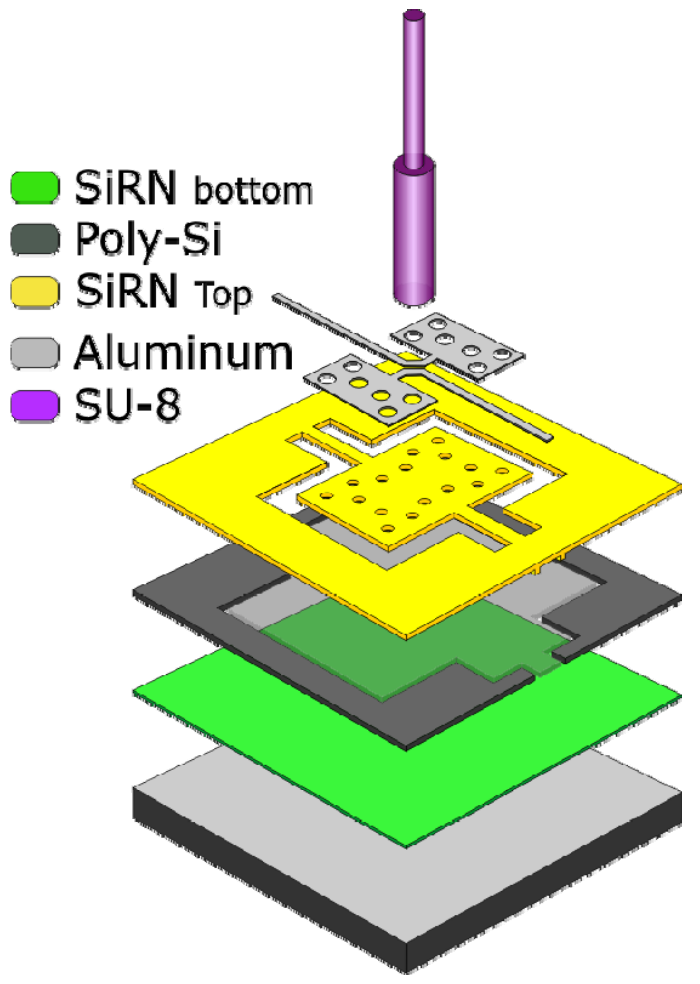

Figure 2. Schematic representation of the single-hair sensor fabrication process

Subsequently, the SU-8 hair is fabricated with two different diameters using a two-step photolithography process. Finally, the flow sensors were released from the substrate by etching of the sacrificial poly-silicon layer. Fig. 2 shows a 3D schematic of the artificial hair flow-sensor geometry. In this design, each hair flow-sensor contains a group of 124 hairs with the same orientation connected in parallel to increase the capacitance changes. The maximum sensitivity axis of each individual hair is aligned with a $45^{\circ}$ tilting angle relative to the long axis of the sensor die, as shown in Fig. 3.

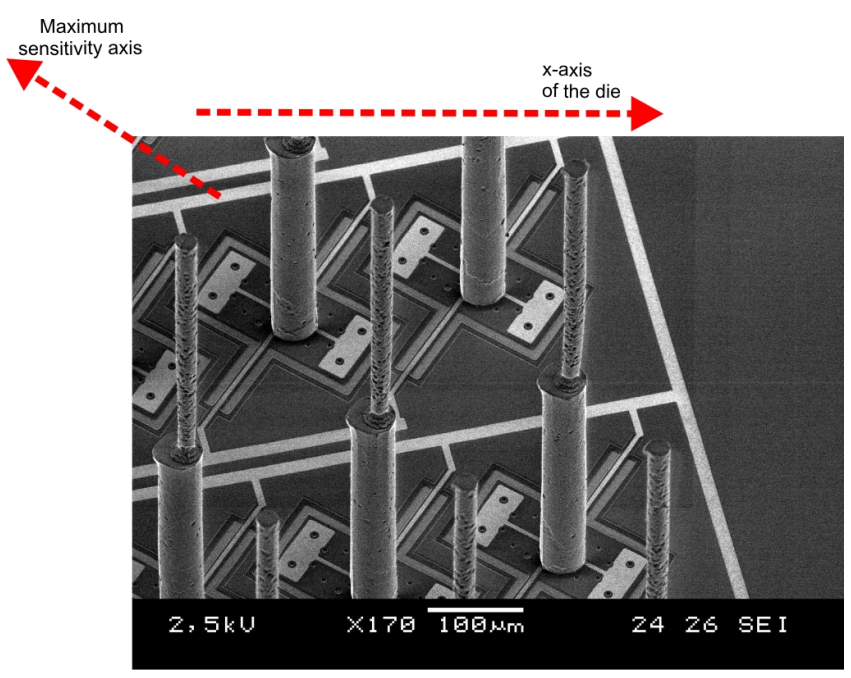

Figure 3. A SEM image for the artificial hair flow-sensor showing the grouping principle.

\section{CURRENT ARTIFICIAL HAIR SENSOR DESIGN}

Previously, we have shown advancements in the fabrication and design of flow-sensitive hair-sensors by improving their mechanical behaviour [6,7]. However, in that sensor design the underlying silicon substrate forms the common electrode of the integrated capacitors. This generates large parasitic capacitances in parallel with the sensing capacitors, as shown in Fig. 4. These parasitics enlarge the noise effect at the sensor-electronics interface. Moreover, the extra parasitics added to the sensing capacitors decrease significantly the fractional capacitance change, which determines the sensitivity. Therefore, to increase the magnitude of these capacitance changes, and hence the SNR, 124 hairs were connected in parallel into one group (i.e. adding the common capacitance changes but averaging out noise from similar but independent hairs). Here we look at improvement of the detection limit by reduction of the parasitic effects inherent to the use of a silicon substrate as common electrode. The use of Silicon-on-Insulator technology allows us to redesign the electrode system of the hair sensor and to largely eliminate parasitic capacitances.

In the current hair-sensor design the carrier signal electrodes are separately defined in the silicon device layer (using deep isolated trenches) while the common electrode for the output signal is implemented by an aluminum layer on top of the membrane. Consequently, the overlap is minimal, assuring reduced parasitic capacitance between carrier signals to- and output signals from the device. To further improve the hair sensor performance, the sensor die is shielded by connecting the inactive areas in the device layer and the carrier wafer to ground assuring minimal parasitics and electromagnetic interference. Fig. 4 shows a representation of 
the equivalent electrical circuit with the parasitic capacitances in both the previous hair sensor design and the current hair sensor design.

The fabrication of the current hair-sensor starts by etching deep isolation trenches into the SOI $25 \mu \mathrm{m}$-device layer down to the isolating $\mathrm{SiO}_{2}$ layer by anisotropic reactive-ion etching (RIE) to form the bottom electrodes. Patterning of the membrane, aluminium electrodes and SU-8 processing have not undergone any changes compared to the fabrication process of the previous sensor design [6]. Fig. 5 illustrates the fabrication scheme of the current hair-sensor design using SOI technology.

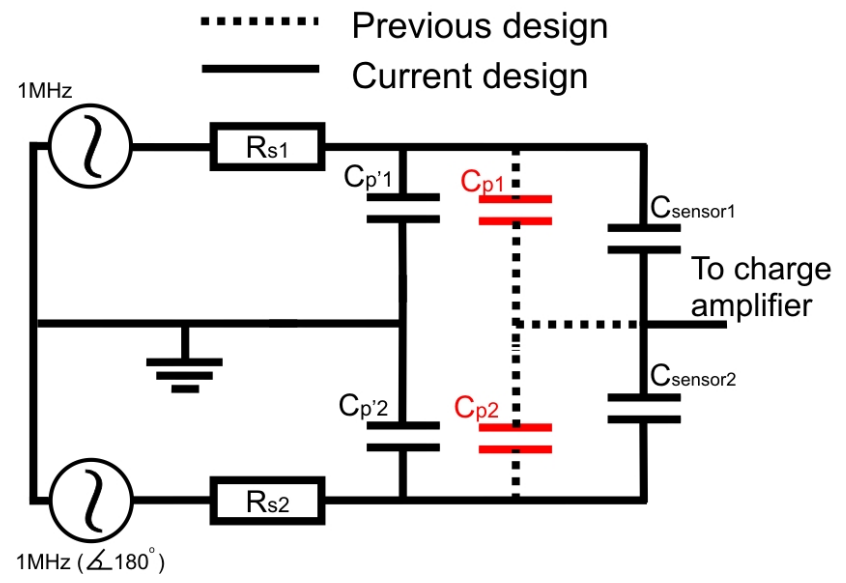

Figure 4. Schematic representing the electrical equivalent circuit at the hair sensor-electronics interface. The dashed lines represent the previous sensor design and the current design is represented by continuous lines.

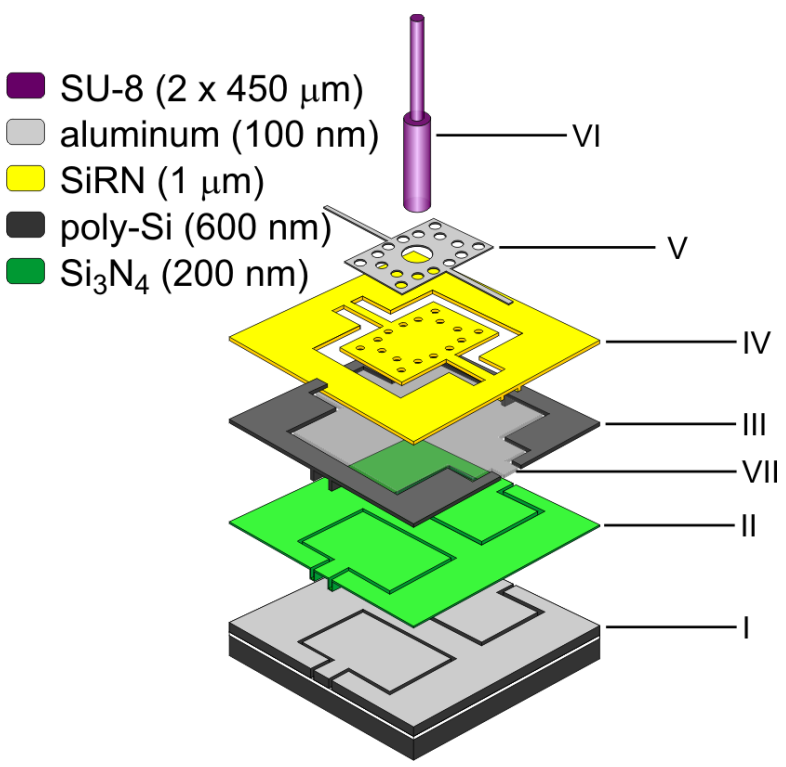

Figure 5. Schematic representation of the current hair-sensor fabrication process.

\section{EXPERIMENTS AND RESULTS}

To determine the improvements of the current SOI technology over the previous sensor-implementation the detection limits of both hair sensor designs were measured and compared. A loudspeaker is used to generate a sinusoidal air flow. The hair sensor was mounted on top of a rotating stage in very near field conditions. Both hair sensors have identical mechanical design, identical frequency response and are measured using the same interfacing electronics. The detection limit for each hair sensor was determined for the direction parallel to the axis of maximum sensitivity.

\section{A. Detection limit}

The new design enables us to measure small capacitance changes and to drastically reduce the number of hairs per device, previously up to 124 sensors in parallel, to ultimately single-hair sensors. Fig. 6 shows the current single-hair sensor illustrating the new electrode design.

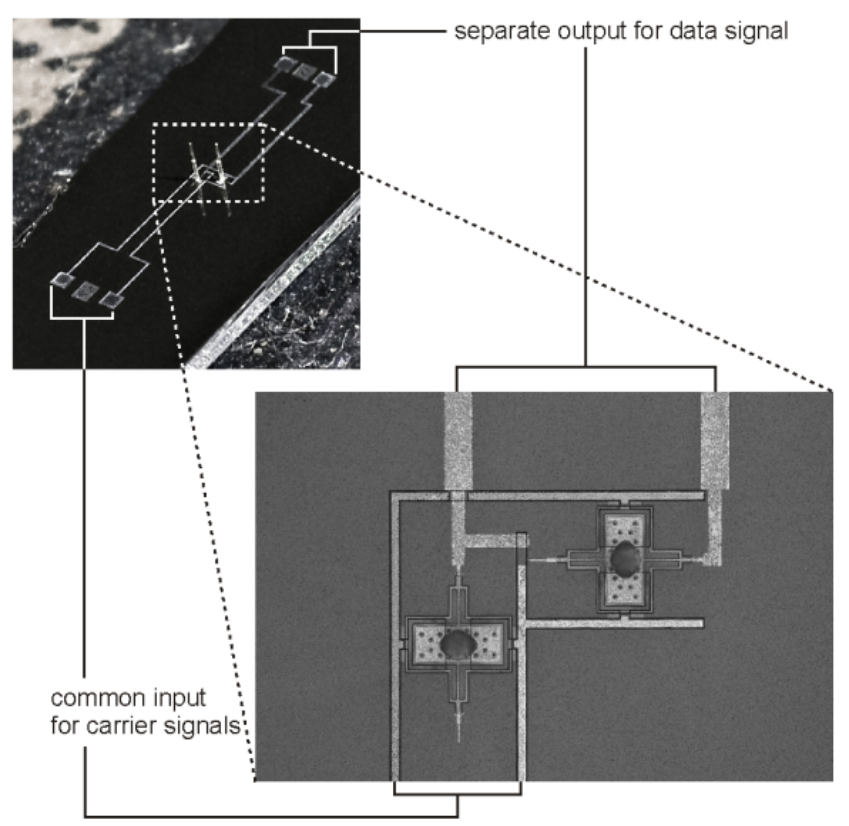

Figure 6. Images for the current single-hair sensor.

The threshold limits of the single-hair sensor (current design) and the hair sensor array (previous design) are defined as the air flow amplitude at which the sensor response has a signal-to-noise ratio (SNR) equal to one. This is indicated at the intersection of the asymptotic sensor response with its noise level. Due to the reduction in parasitic capacitance the single-hair sensor shows better response threshold down to 1 $\mathrm{mm} / \mathrm{s}$ air-flow amplitude as measured in a bandwidth of 3 $\mathrm{kHz}$. Fig. 7 shows an example of the voltage response for both hair sensors as function of air-flow amplitude oscillating at $250 \mathrm{~Hz}$. Based on these results, the detection limit of the new single hair sensor was enhanced by $52 \%$ down to $1 \mathrm{~mm} / \mathrm{s}$ relative to the previous design consisting of 124 hair-sensors in parallel. 


\section{B. Directivity}

Fig. 8 shows the single hair sensor response as it is rotated over $360^{\circ}$ with $10^{\circ}$ steps. In addition to the improvement in the detection limit, the current single-hair sensor shows an almost textbook like figure of eight directivity. This can be related to the elimination of viscous coupling between the hairs and asymmetric flow disturbances generated by array effects due to the grouping of the hairs on one die.

Since we are able now to measure capacitance changes from a single-hair sensor, whereby it is unnecessary to average over a large area of the die, it becomes feasible to determine flow phenomena at relative small scales as far as viscous coupling allows.

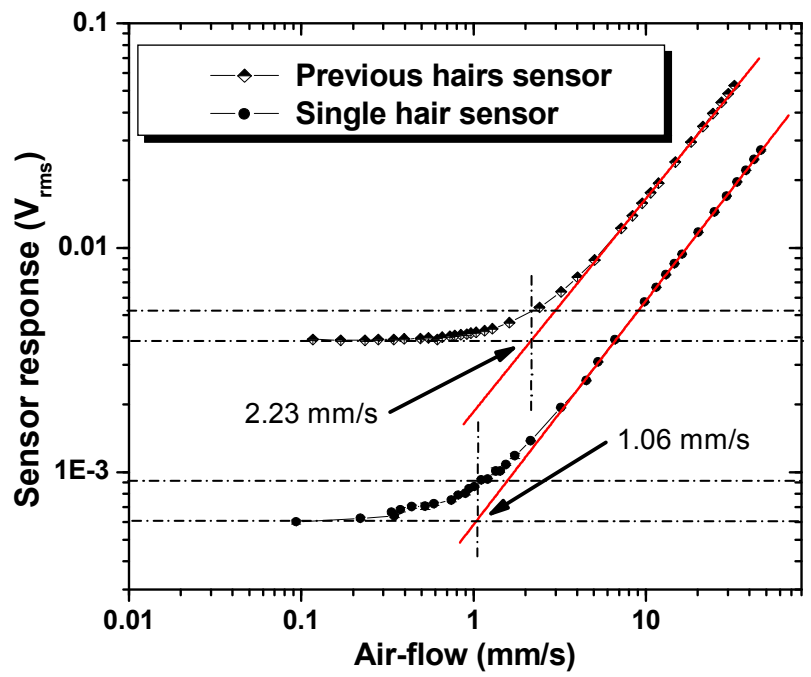

Figure 7. Voltage response of both the old hair sensor and the single-hair sensor as function of flow velocity amplitude at $250 \mathrm{~Hz}$ plotted together with the asymptotic lines (in red) to determine the threshold flow amplitude.

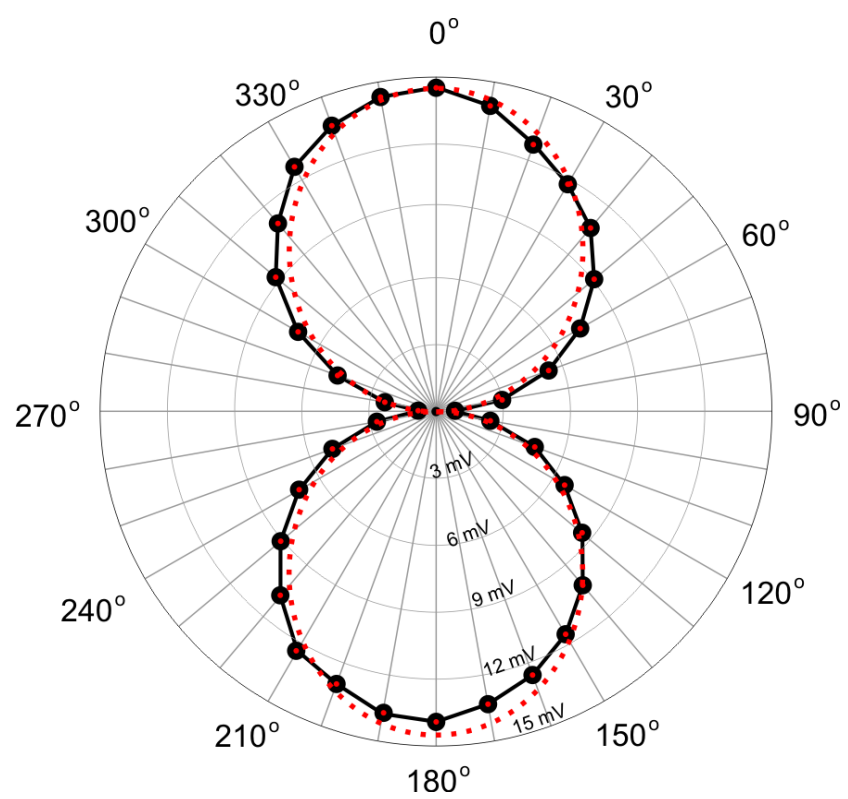

Figure 8. Directivity measurement of the single-hair sensor (black lines and dots) compared to an ideal figure of eight (red dashed line).

\section{CONCLUSIONS}

We have shown the improvement in detection limit of our artificial hair flow-sensors resulting from a better sensor electrode design. Taking advantage of deep isolation trenches to define electrode areas, SOI technology opens possibilities to fabricate arrays of single-hair sensors. Future work will focus on further reduction of electronic noise which is currently determining the detection limit of the hair sensors. In combination with efficient array addressing techniques the current technology allows for high-resolution spatio-temporal flow pattern observations, so as to form the equivalent of a flow-camera.

\section{ACKNOWLEDGMENT}

The authors want to thank our colleagues in the EU project CILIA (www.cilia-bionics.org) for stimulating discussions and input to this work.

\section{REFERENCES}

[1] T. Shimozawa, T. Kumagai, and Y. Baba, "Structural scaling and functional design of the cercal wind-receptor hairs of crickets", Journal of Comparative Physiology - A Sensory, Neural, and Behavioral Physiology, 183, 1998, pp. 171-186.

[2] S. Dijkgraaf, "The functioning and significance of the lateral-line organs", Biological reviews of the Cambridge Philosophical Society, 38, 1963, pp. 51-105.

[3] W. Gnatzy and R. Heusslein, "Digger wasps against crickets. I. Receptor involved in the antipredator strategies of the prey", Naturwissenschaften, 73, 1986, pp. 212-215.

[4] C. Magal, O. Dangles, P. Caparroy, J. Casas, "Hair canopy of cricket sensory system tuned to predator signals", Journal of Theoretical Biology, 241 (3), 2006, pp. 459-466.

[5] G. A. Jacobs, J. P. Miller, Z. Aldworth, "Computational mechanisms of mechanosensory processing in the cricket", Journal of Experimental Biology, 211, 2008, pp. 1819-1828.

[6] C.M. Bruinink, R.K. Jaganatharaja, M.J. de Boer, J.W. Berenschot, M.L. Kolster, T.S.J. Lammerink, R.J. Wiegerink, G.J.M. Krijnen, "Advancements in technology and design of biomimetic flow-sensor arrays" In: 22nd IEEE International Conference on Micro Electro Mechanical Systems, Italy, 2009, pp. 152-155.

[7] G.J.M. Krijnen et al.,"MEMS based hair flow-sensors as model systems for acoustic perception studies", Nanotechnology, 17, 2006, pp. S84S89. 